\title{
Imaging of Hepatocellular Carcinoma by Computed Tomography and Magnetic Resonance Imaging: State of the Art
}

\author{
Christoph J. Zech Maximilian F. Reiser Karin A. Herrmann \\ Institute of Clinical Radiology, University Hospitals - Grosshadern, Ludwig Maximilians University, \\ Munich, Germany
}

\section{Key Words}

Liver cirrhosis $\cdot$ Hepatocellular carcinoma $\cdot$ Regenerative nodule $\cdot$ Magnetic resonance imaging $\cdot$ Computed

tomography

\begin{abstract}
Hepatocellular carcinoma (HCC) is a very frequent tumor worldwide. Its incidence is linked to the distribution of liver cirrhosis and viral hepatitis, which are the main risk factors for the development of HCC. For the evaluation of the cirrhotic liver and for the diagnosis of HCC, multidetector computed tomography (MDCT) proved to be a robust and reliable tool. In MDCT the diagnosis of HCC can be made based on neovascularization with increased arterial and decreased portal venous supply. With modern magnetic resonance imaging (MRI), spatial resolution and robustness increased dramatically. Beside the evaluation of neovascularization by means of gadolinium-enhanced early dynamic MRI, the main advantages of MRI are additional information on tissue composition and liver-specific function. With diffusionweighted imaging or plain $\mathrm{T}_{1}$ - and $\mathrm{T}_{2}$-weighted sequences, different tissue elements like fat, hemorrhage, glycogen, edema and cellular density can be evaluated. Liver-specific contrast agents give insight into the Kupffer cell density or the hepatocellular function. The integration of all these parts into the MR examination allows for a very high detection rate for overt HCC nowadays, although very small HCCs are still a
\end{abstract}

challenge. Moreover, insight into the different stages of hepatocarcinogenesis can be possible with MRI. Despite its limited availability in some countries, it has to be rendered to be the modality of choice for the distinct evaluation of the cirrhotic liver.

Copyright $\odot 2009$ S. Karger AG, Basel

\section{Introduction}

Hepatocellular carcinoma (HCC) is among the most frequent tumors worldwide with an incidence rate of 20$150 / 100,000 / y e a r$ in high-risk areas in Asia and Africa, of $5-20 / 100,000 / y e a r$ in the areas with intermediate risk in Japan and the Mediterranean countries and of 5/100,000 and less in areas with low risk in Northern Europe and the USA [1]. One of the major risk factors for the development of $\mathrm{HCC}$ is chronic hepatitis $\mathrm{B}$ and $\mathrm{C}$ and chronic alcohol abuse, especially if liver cirrhosis is already present. In case of underlying liver cirrhosis, a stepwise development of carcinogenesis from areas of regeneration to overt HCC has been described. According to this concept, the most common terminology of the International Working Party of the World Congress of Gastroenterology defined regenerative nodules, low-grade dysplastic nodules, high-grade dysplastic nodules and well-differentiated HCC as steps from regeneration to cancer [2-4]. However, also a de novo development of HCC takes place 
Fig. 1. CT scan of a 62-year-old female patient with liver cirrhosis Child-Pugh A based on a chronic hepatitis C. In liver segment VIII, a $2.3-\mathrm{cm}$ lesion with strong arterial supply (a) and rapid washout (b) was detected. Note the corona enhancement in the portovenous scan (b), which is typical for HCC. The lesion was diagnosed noninvasively as HCC based on the EASLAASLD criteria. The MR images of the same patient are shown in figure 5 .
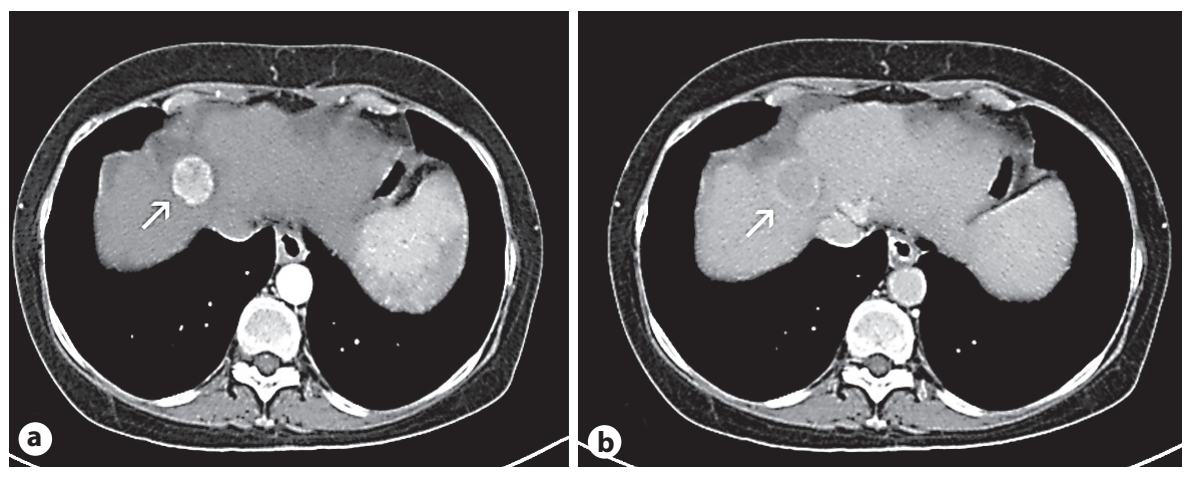

[2]. The challenge for the gastroenterologist, hepatic surgeon and radiologist is to detect premalignant and malignant lesions early, to distinguish between the different regenerative nodules and HCC in patients with liver cirrhosis and to allocate the patients with HCC properly to the treatment options which are nowadays available.

\section{Computed Tomography}

Computed tomography (CT) has developed dramatically with the introduction of the multidetector technology (MDCT). Especially the abdomen, where motion artifacts due to respiratory motion and bowel peristalsis are disturbing, takes great advantage from this technique.

Adequate examination technique is critical for sensitive detection of HCC. A triphasic examination of the liver with a plain, a late-arterial (arterial-dominant) and a portovenous phase scan can be regarded as standard today. The value of delayed scans (e.g. 3-5 min after contrast agent injection) to depict pathological tumor washout has been demonstrated in the literature [5]. With the short acquisition times of MDCT, contrast agent timing has become critical, since the optimal enhancement phase has to be included within a very short acquisition window. Therefore, the use of modern contrast agent power injectors and bolus timing is mandatory [6]. Depending on the iodine concentration, fast flow rates up to 5 or $6 \mathrm{ml} / \mathrm{s}$ are recommended [6]. The dosing of the contrast agent should be related to the body weight with $1.5-$ $2 \mathrm{ml} / \mathrm{kg}$ b.w. (for a concentration of $300 \mathrm{mg}$ iodine/ml) [7]. The delay between reaching the triggering threshold of $100 \mathrm{HU}$ in the aorta and starting the scan is usually $15 \mathrm{~s}$ for the late-arterial (or arterial-dominant) phase. The optimal slice thickness of reconstructed CT images of the liver is still under debate. Past publications have been quite restricted here and recommended that the slice thickness should not be $<5 \mathrm{~mm}$ for the low-contrast organ liver $[8,9]$. Recent publications, however, disocvered advantages for a reconstructed slice thickness down to $3 \mathrm{~mm}$ [10]. If the slice thickness is further decreased, image noise and low contrast overwhelm the positive effect of geometrical resolution. This phenomenon might only be covered with inadequately high radiation doses.

For imaging of HCC and lesions in the cirrhotic liver the main diagnostic criterion in $\mathrm{CT}$ is the depiction of changes in the vascular supply of liver nodules due to neoangiogenesis as described for CT during hepatic angiography (CTHA) by Matsui [4]. In addition to the intrahepatic staging with regard to number and size of HCC lesions, complicating factors like liver cirrhosis and patency of the portal vein are also of relevance for assigning patients to a proper treatment regimen.

In case of intravenous contrast application, HCC typically presents as a hyperdense lesion in the arterial-dominant phase with following washout to iso- or mostly hypodensity in the portovenous phase (fig. 1). The presence of pathological washout can be depicted with higher accuracy in delayed phases $[5,11]$. This is of importance since both the presence of hypervascularity and pathological washout with one or two imaging techniques in lesions ranging from 1 to $2 \mathrm{~cm}$ and $>2 \mathrm{~cm}$, respectively, allows the definite diagnosis of a HCC non-invasively according to the EASL-AASLD practice guidelines [12]. In CT the depiction of these vascular changes is crucial since there are no other reliable criteria to detect or characterize HCC (fig. 2). The lack of additional criteria is the main reason for false-positive findings (e.g. arteriovenous shunts, dysplastic nodules with pathological vascularization) or false-negative findings (e.g. well-differentiated HCC without arterial hyperenhancement, hypovascular HCC). From a radiological/pathological aspect, the 
Fig. 2. CT scan of a hypovascular HCC in a 75-year-old male patient with liver cirrhosis Child-Pugh A based on a chronic hepatitis B. In liver segment II, a hypodense, well-circumscribed round lesion (arrow) is seen in plain CT (a). In the arterial-dominant phase (b), only faint enhancement can be delineated, overall the lesions remains hypodense in arterial and portovenous phase (c). The patient underwent angiography, which also did not reveal a hypervascular tumor (not shown here). In the plain CT after probatory segmental lipiodol injection (d) during angiography, the lesion still appears hypodense. Percutaneous biopsy revealed an undifferentiated HCC (WHO grade 3). The patient was treated with radiofrequency ablation.
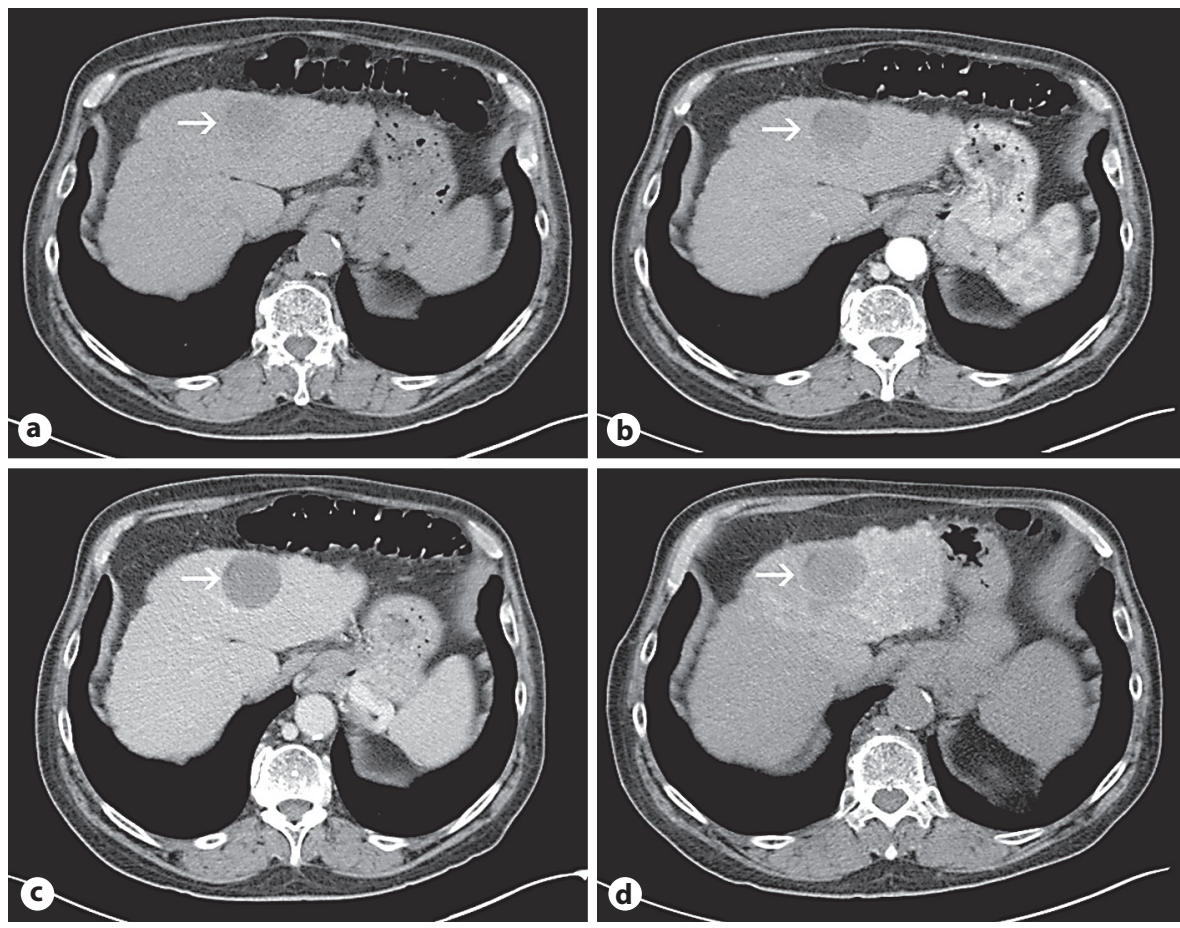

differentiation between regenerative nodules, low-grade and high-grade dysplastic nodules and well-differentiated HCC would be desirable. This so-called 'grey zone of hepatocarcinogenesis' remains still unclear for imaging (either with CT or magnetic resonance imaging (MRI)) as well as even sometimes for pathology [13]. MDCT as a modality which works based on attenuation differences due to a different vascular supply is with current technology as well as mere extracellular MRI not able to contribute to this differential diagnosis with confidence [14].

The detection rates of HCC reported in the literature are highly variable. One trial with a very stringent methodology and whole-liver explant correlation showed a sensitivity of $61 \%$, a specificity of $66 \%$ and a negative predictive value of $30 \%$ for the detection of HCC by means of triphasic CT [14]. A subgroup analysis in this trial revealed a strong influence of the lesion size. While lesions $>2 \mathrm{~cm}$ were detected in $100 \%$, lesions $<1 \mathrm{~cm}$ were only detected in 10\% [14]. Another trial with a 4-row MDCT demonstrated an overall sensitivity of $73 \%$ for HCC detection, with also markedly reduced detection rates (33\%) for lesions $<1 \mathrm{~cm}$ [8]. A recent publication by Maetani et al. [15] exhibited a sensitivity, positive predictive value and accuracy for HCC detection by means of MDCT with 87, 96 and 84\%, respectively. Kim et al. [16] evaluated ferucarbotran-enhanced 3.0-T MRI versus triple-phase MDCT and found a superior sensitivity for HCC detection of $98.1 \%$ in MRI versus $92.9 \%$ in MDCT. The higher sensitivity of MRI was largely attributable to a greater ability of the MRI to detect small $(<1 \mathrm{~cm})$ HCC $(92.6 \%$ for MRI and $37.0 \%$ for MDCT).

Since CT can only depict the vascularity of lesions, it is difficult to distinguish between simple regenerative nodules, high-grade dysplastic nodules and early HCC in the cirrhotic liver $[13,14]$. The advantages of MRI in this respect are the possibility of tissue characterization based on different contrast weightings of the pulse sequences $\left(\mathrm{T}_{1}, \mathrm{~T}_{2}\right)$ and the availability of several liver-specific contrast agents - as will be pointed out in the following part of this article.

\section{Magnetic Resonance Imaging}

The evaluation of the cirrhotic liver is a challenging task for every imaging modality and this holds also true for MRI. However, with MRI the criteria for the evaluation of the focal lesions in cirrhotic liver are expanded from vascularity alone to cellular density and tissue composition by means of precontrast sequences and diffu- 
sion-weighted MRI (DWI), to the presence of Kupffer cells by means of superparamagnetic particles of iron oxide (SPIO) and to the integrity of hepatocellular function and biliary excretion by means of hepatobiliary contrast agents (table 1).

MRI has made dramatic changes with regard to artifact robustness, spatial resolution and speed in the abdominal area. The use of phased-array multichannel coils and fast imaging techniques like gradient recalled echo (GRE) or fast spin echo (FSE) techniques are now established since many years as a standard for abdominal MRI. The introduction of parallel imaging, DWI, 3D GRE techniques with interpolation and ultrashort repetition times and the navigator techniques of respiratory triggering have been introduced recently and are by now already in broad use [17-19]. A liver MR study usually comprises a $\mathrm{T}_{1} \mathrm{w}$ 2D GRE sequence in-phase and opposed-phase, a $\mathrm{T}_{2} \mathrm{~W}$ single-shot FSE and/or $\mathrm{T}_{2} \mathrm{~W}$ multi-shot FSE with fat saturation, a DWI echoplanar imaging sequence and a dynamic $\mathrm{T}_{1} \mathrm{w}$ 3D GRE sequence with fat saturation after contrast agent injection. Depending on the type of contrast medium, additional sequences for the liver-specific phase are performed, as $\mathrm{T}_{2 * \mathrm{~W}}$ GRE sequences for SPIOenhanced MRI and $\mathrm{T}_{1} \mathrm{~W} 2 \mathrm{D}$ or $3 \mathrm{D}$ sequence for hepatobiliary agents.

The contrast agents used for liver MRI are on the one hand extracellular, unspecific gadolinium agents, and on the other, liver-specific contrast agents. The latter can be divided into iron-oxide particles (SPIO), which are targeted to the reticuloendothelium system, to the so-called Kupffer cells and the hepatobiliary contrast agents, which are targeted directly to the hepatocyte and are excreted via the bile. There are currently five approved liver-specific contrast agents with different availabilities on the market: ferumoxide (Endorem ${ }^{\circledR}$, Guerbet, Aulnay-sousBois, France); ferucarbotran (Resovist ${ }^{\circledR}$, Bayer Schering Pharma AG, Berlin, Germany); mangafodipir trisodium/Mn-DPDP (Teslascan ${ }^{\circledR}$, GE Healthcare Biosciences, Little Chalfont, UK); gadobenate-dimeglumine/GdBOPTA (MultiHance ${ }^{\circledR}$, Bracco Imaging, Milan, Italy), and gadoxetic-acid/Gd-EOB-DTPA (Primovist ${ }^{\circledR}$, Bayer Schering Pharma AG, Berlin, Germany).

Non-enhanced MRI plays an important role in the characterization of different tissue components. The signal intensity of hepatic nodules in the cirrhotic liver can vary in $\mathrm{T}_{1} \mathrm{w}$ and $\mathrm{T}_{2} \mathrm{~W}$ plain sequences. It has been demonstrated that copper deposition, glycogen, intratumoral bleeding or fat within a nodule causes hyperintensity on plain $\mathrm{T}_{1} \mathrm{w}$ sequences [20]. Since hyperintensity occurs in dysplastic nodules as well as in approximately one third

Imaging of HCC by CT and MRI:

State of the Art
Table 1. Key features of regenerative nodules, dysplastic nodules and HCCs in MRI

\begin{tabular}{|c|c|c|c|}
\hline & $\begin{array}{l}\text { Regenerative } \\
\text { nodules }\end{array}$ & $\begin{array}{l}\text { Dysplastic } \\
\text { nodules }\end{array}$ & Overt HCCs \\
\hline \multicolumn{4}{|l|}{ Plain MRI } \\
\hline $\mathrm{T}_{1} \mathrm{w} 2 \mathrm{D}$ GRE & $=$ & $\uparrow$ & $\downarrow$ or $\uparrow$ \\
\hline $\mathrm{T}_{2} \mathrm{w}$ FSE fs & $=$ or $\downarrow$ & $=$ or $\downarrow$ & $\uparrow$ \\
\hline \multicolumn{4}{|c|}{ Early dynamic phase } \\
\hline Arterial phase & $\begin{array}{l}\text { no } \\
\text { enhancement }\end{array}$ & $\begin{array}{l}\text { no } \\
\text { enhancement }\end{array}$ & $\begin{array}{l}\text { arterial } \\
\text { enhance- } \\
\text { ment }\end{array}$ \\
\hline $\begin{array}{l}\text { Portovenous/ } \\
\text { delayed phase }\end{array}$ & $\begin{array}{l}\text { no } \\
\text { washout }\end{array}$ & $\begin{array}{l}\text { no } \\
\text { washout }\end{array}$ & $\begin{array}{l}\text { venous } \\
\text { washout }\end{array}$ \\
\hline \multicolumn{4}{|l|}{ Liver-specific phase } \\
\hline $\begin{array}{l}\text { SPIO } \\
\text { Hepatobiliary }\end{array}$ & $=$ or $\downarrow$ & $=$ & $\uparrow$ \\
\hline agents & $=$ & $\downarrow$ or $\uparrow$ & $\downarrow$ \\
\hline
\end{tabular}

This table summarizes the most important and typical MRI findings in regenerative nodules, dysplastic nodules and HCCs. However, this table is not able to express the complexity of all kinds of borderline lesions (e.g. high-grade dysplastic nodule vs. early HCC) and does not consider atypical or infrequent imaging findings (like hepatobiliary accumulation in well-differentiated HCC or hypovascular HCC).

of moderately differentiated HCC, it seems impossible to distinguish the nature of a hepatic nodule based on $\mathrm{T}_{1} \mathrm{w}$ signal alone [21] (fig. 3). For the signal behavior in $\mathrm{T}_{2} \mathrm{~W}$ sequences, hyperintensity with depiction of a mosaic pattern has been described to be typical for HCC and has been seen in $77 \%$ of cases $>3 \mathrm{~cm}$ [21]. In the same evaluation, overall $91 \%$ of HCC depicted as hyperintense lesions in $\mathrm{T}_{2} \mathrm{~W}$ images, whereas the signal intensity in $\mathrm{T}_{1} \mathrm{~W}$ sequences was quite equally distributed between hypo-, iso- and hyperintense [21]. $6 \%$ isointense $\mathrm{T}_{2} \mathrm{~W}$ nodules represented well-differentiated HCCs (grade 1), all 3\% hypointense nodules were found to be necrotic HCC [21]. Based on these data, the combination of signal intensity on $\mathrm{T}_{1} \mathrm{w}$ and $\mathrm{T}_{2} \mathrm{w}$ images is rendered to be a useful adjunct in the differential diagnosis of hepatocellular nodules in cirrhotic livers. A nodule that is hyperintense on $\mathrm{T}_{1} \mathrm{w}$ images and isointense on $\mathrm{T}_{2} \mathrm{~W}$ images usually indicates that the lesion bears a certain risk for ongoing malignant transformation (often high-grade dysplastic nodules or early well-differentiated HCC). In contrast, nodules that are hyperintense on $\mathrm{T}_{1} \mathrm{w}$ images and iso- or hyperintense on $\mathrm{T}_{2} \mathrm{~W}$ images usually represent an overt HCC [22] 
Fig. 3. Plain MR scans of two different patients with liver cirrhosis. In the upper row (a, b), a nodule is depicted which exhibits hyperintensity in the plain $\mathrm{T}_{1} \mathrm{w} 2 \mathrm{D}$ GRE sequence in-phase (a), and hypointensity in the plain $\mathrm{T}_{2} \mathrm{w}$ FSE with fat saturation (b). No pathological vascularization was depicted (not shown here). Based on follow-up the nodule was diagnosed as a regenerative nodule. The nodule in the lower row $(\mathbf{c}, \mathbf{d})$ is also depicted with hyperintensity in the $\mathrm{T}_{1} \mathrm{w}$ scan (c), but already the size of the lesion and the pseudocapsule raise suspicion. This nodule appears hyperintense on the $\mathrm{T}_{2} \mathrm{~W}$ image (d) with a mosaic pattern. The histological diagnosis of the nodule is HCC (same lesions as shown in fig. 2).
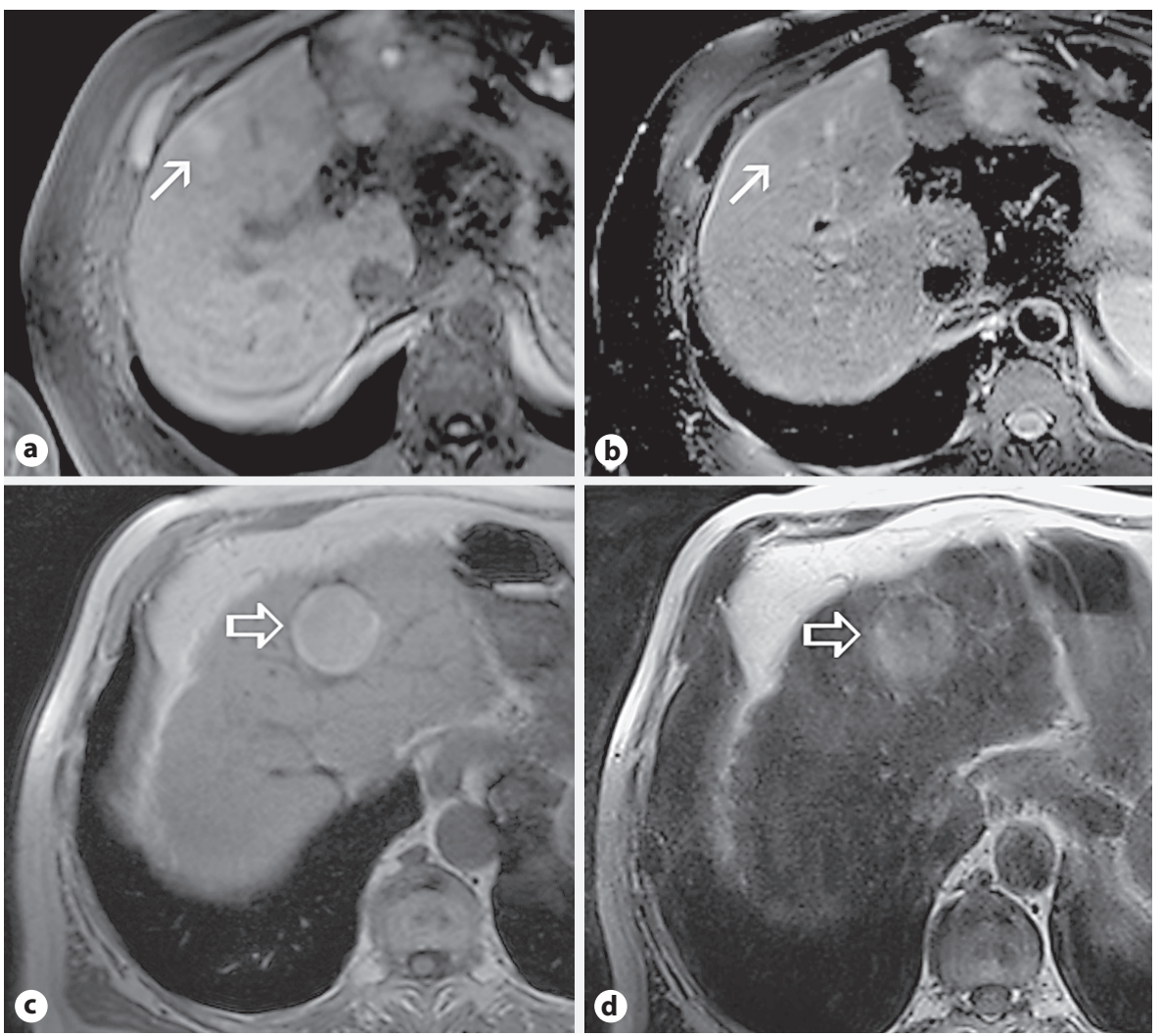

(fig. 3c, d). A nodule being isointense on $\mathrm{T}_{1} \mathrm{w}$ images and hypo- or isointense on $\mathrm{T}_{2} \mathrm{~W}$ images exhibits the typical signal behavior of a regenerative nodule [23] (fig. 4). Other typical morphological features of HCC that can be seen on precontrast (and contrast-enhanced) MR images include a pseudocapsule [24] and a mosaic pattern [21, 25].

DWI has long played only a minor role in abdominal imaging; however, with new scanner generations with homogenous magnetic fields and with the introduction of parallel imaging, DWI with echoplanar images is feasible with a high image quality and robustness $[26,27]$. DWI can help to increase the detection rate of focal liver lesions, especially due to the black-blood effect, which helps to perceive even very small lesions or lesions directly adjacent to vessels easily and fast [27]. A recent study that focused on the added value of DWI in the cirrhotic liver showed that the detection rate for HCC was increased from $83-85$ to $98 \%$ in a two-reader analysis of gadolinium-enhanced images only and gadolinium-enhanced + DWI images [28]. Moreover, the quantification of restricted diffusion with the apparent diffusion coefficient helps to differentiate between benign and malig- nant lesions [26]. However, up to now there is no evidence in the literature in how far DWI might be a feasible approach to differentiate between regenerative nodules, dysplastic nodules and HCC. Another interesting application for DWI in the cirrhotic liver is the quantification of liver fibrosis in chronic hepatitis, which shows very promising results in the literature [29].

The dynamic MR examination with gadoliniumbased contrast agents provides information on the changes of vascular supply within different hepatic nodules in the cirrhotic liver and is a crucial part of the evaluation of patients with suspected HCC (fig. 5). With regard to the criteria for the diagnosis of overt HCC, the same contrast agent behavior as described for CT is used for MRI, which means hypervascularity in the arterial-dominant phase and pathological washout in the portovenous or delayed phase. Gadolinium-enhanced MRI with $\mathrm{T}_{1} \mathrm{w} 3 \mathrm{D}$ GRE sequences, allowing for thin slices, enables detection of HCC nodules with $76 \%$ sensitivity, $75 \%$ specificity and a negative predictive value of 50\% [14]. The reported values of Burrel et al. [14] may seem only moderate; however, it has to be taken into account that they were validated against whole-liver explant specimens and that a 

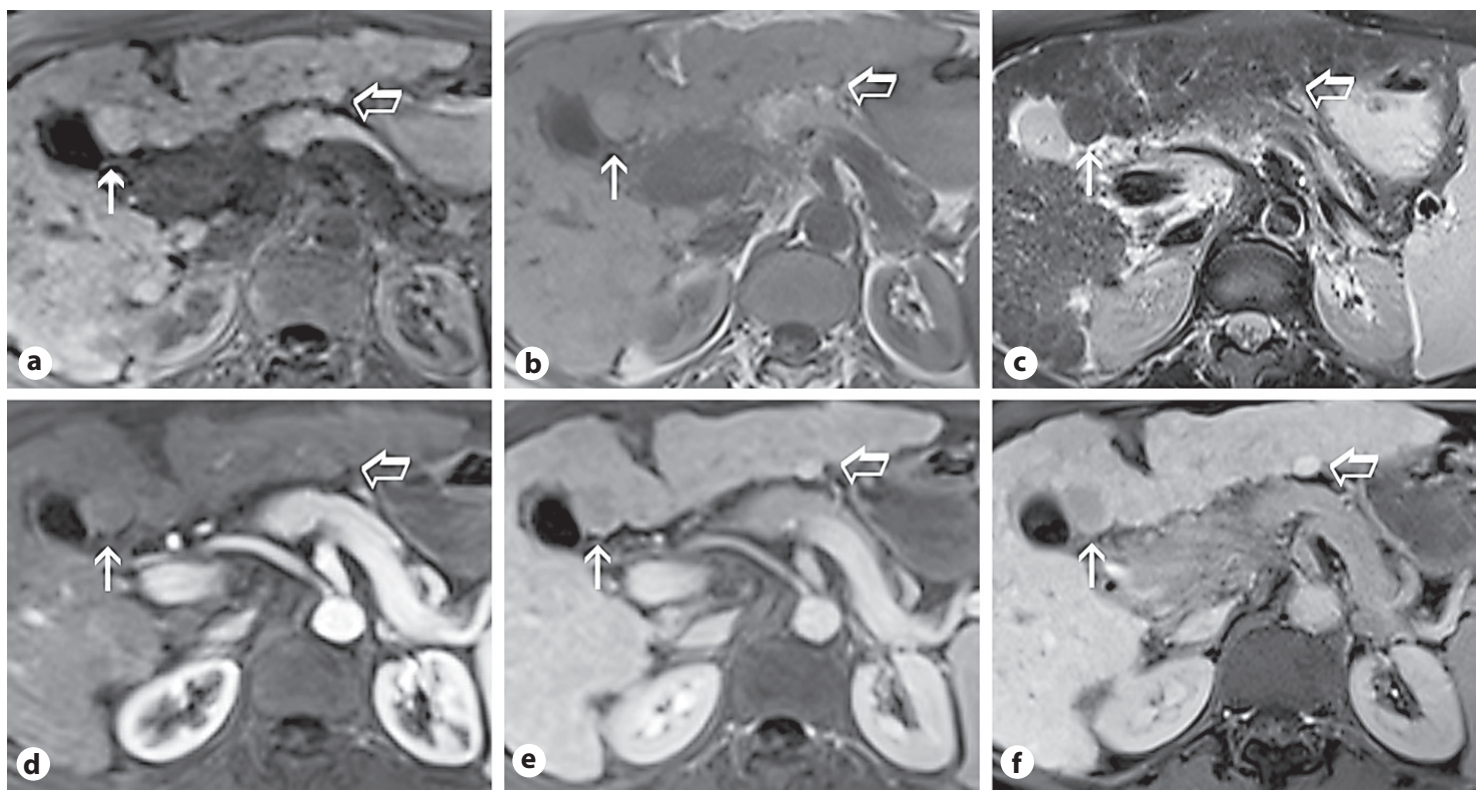

Fig. 4. MR scan of a 45-year-old female patient with liver cirrhosis due to chronic hepatitis C. The patient suffers from overt HCC (not shown here). Additionally, two nodules were detected in segment IVb (small arrow) and in segment III (broad arrow). The nodule in segment IVb is slightly hyperintense in the $\mathrm{T}_{1} \mathrm{w} 3 \mathrm{D}$ GRE with fat saturation (a) and $\mathrm{T}_{1} \mathrm{w} 2 \mathrm{D}$ GRE without fat saturation (b) and isointense in the $\mathrm{T}_{2} \mathrm{w}$ FSE sequence (c). In the $\mathrm{T}_{1} \mathrm{w}$ 3D GRE sequence after Gd-EOB-DTPA injection (d), no hypervascularity can be seen in the arterial phase (considering the precontrast signal); however, the nodule displays faint washout in the portovenous scan (e) and decreased hepatobiliary enhancement in the liver-specific phase $20 \mathrm{~min}$ after injection (f). This pattern is compatible with a high-grade dysplastic nodule. On the other hand, the smaller nodule in segment III is nearly isointense in the $\mathrm{T}_{1} \mathrm{w}$ images $(\mathbf{a}, \mathbf{b})$ and hypointense in the $\mathrm{T}_{2} \mathrm{w}$ FSE sequence $(\mathbf{c})$. In the arterial phase no hypervascularity can be seen and no washout in the portovenous scan (e). There is some accumulation of contrast agent in the liver-specific phase. This pattern is compatible with a normal regenerative nodule. Due to the dysplastic nodule in segment III, the patient is in close follow-up; since 9 months both lesions are unchanged. large number of small lesions $(<2 \mathrm{~cm})$ were included. Overall, MRI showed superior results to spiral CT in the detection of HCC in this study (sensitivity $76 \%$ MRI vs. $61 \% \mathrm{CT}$ ). For HCC $>2 \mathrm{~cm}, \mathrm{MRI}$ and $\mathrm{CT}$ showed a detection rate of $100 \%$, for $\mathrm{HCC}$ between 1 and $2 \mathrm{~cm} \mathrm{MRI} \mathrm{and}$ CT showed a detection rate of 89 and $65 \%(\mathrm{p}=0.03)$, respectively, and for HCC $<1 \mathrm{~cm}$ values of 34 and $10 \%$ (p = 0.06 ) were seen, respectively. Beside the clear superiority of MRI versus CT for the detection of HCC in the contrast-enhanced dynamic phase examination, these data show that the real challenge for the future and for new techniques will be to increase the detection rate for very small HCC nodules. These results are supported by Lauenstein et al. [30] who found an overall detection rate for HCC with gadolinium-enhanced 3D GRE sequences of $77.8 \%$ ( $>2 \mathrm{~cm} 100 \%,<2 \mathrm{~cm} 55.6 \%$ ). In various other trials, ultrasound, biphasic spiral CT and MRI were compared and MRI proved to be superior to the other modalities in the detection of HCC [31-34]. Beside the high- er detection rate of MRI, it is also considered to be more specific with less false-positive lesions than CT in the differentiation between HCC and regenerative nodules [14, 35]. This can be explained by the additional diagnostic criteria (e.g. $\mathrm{T}_{2} \mathrm{~W}$ signal intensity) for the diagnosis of HCC in MRI (fig. 6). In this respect the results of Holland et al. [36] are interesting; they evaluated lesions seen only on arterial phase images (isointense in $\mathrm{T}_{2} \mathrm{~W}$ images, no washout) and found that $93 \%$ were not HCC at histology. Therefore, the interpretation of hypervascular lesions in the cirrhotic liver has to include also plain MR signal behavior and the washout kinetics.

The use of liver-specific contrast agents aims to increase the sensitivity and specificity of MRI in the cirrhotic liver. With regard to the question whether gadolinium-enhanced MRI or SPIO-enhanced MRI as standalone technologies are superior for HCC detection, the reports in the literature clearly indicate that for detection a gadolinium-enhanced $\mathrm{T}_{1} \mathrm{w}$ dynamic examination re- 
Fig. 5. MR scan of a 62-year-old female patient with liver cirrhosis Child-Pugh A based on a chronic hepatitis $C$. $T_{1} w$ GRE sequence with fat saturation in arterial (a), portovenous (b) and equilibrium phase (c) after bolus injection of Gd-EOB-DTPA; d shows the liver-specific phase 20 min after injection. Note the lesion (arrow) with the typical enhancement pattern of a HCC with arterial hypervascularity and rapid washout, which can be appreciated best in the equilibrium phase scan (c). As an additional criterion for a HCC, missing hepatobiliary uptake with high tumor-to-liver contrast is seen in the liver-specific phase (d). Note multiple small nodules $(<5 \mathrm{~mm})$ with hepatobiliary uptake representing regenerative nodules. The corresponding CT images are shown in figure 1.

Fig. 6. A 44-year-old male patient with a history of alcohol-induced liver cirrhosis and two HCC nodules (arrows). Gd-EOBDTPA-enhanced MRI (a, b) and CT $(\mathbf{d}, \mathbf{e})$ images in arterial and portovenous phase. Note the superior visualization of arterial hypervascularization and especially the better depiction of the washout in MRI (b) versus CT (e). Moreover, with MRI, additional criteria like the liver-specific phase showing missing hepatobiliary uptake (c) and diffusion-weighted images (f) allow a fast and easy detection of lesions.
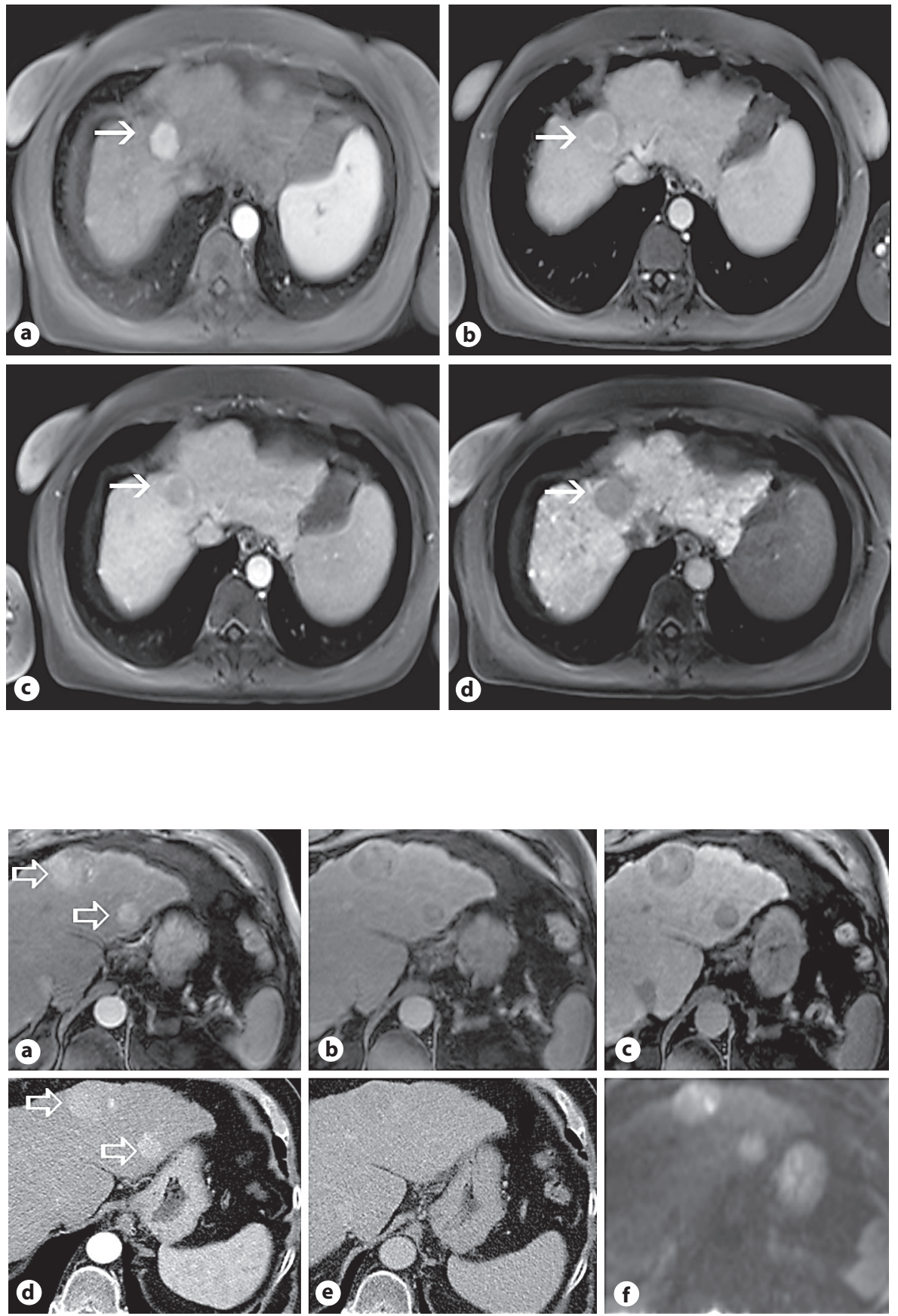

mains the method of choice. In a study by Kim et al. [37] superiority of gadolinium-enhanced MRI over SPIO MRI ( 91.3 vs. $77.3 \%$, respectively) was demonstrated. On the other hand, severe liver cirrhosis with extensive fibrosis may lead to an overestimation of tumor extension in SPIO-enhanced MRI since fibrotic areas do not take up SPIO so that differentiation from HCC nodules may be difficult. SPIO-enhanced MRI helps to differentiate between regenerative nodules, dysplastic nodules and overt HCC based on the different degree of iron uptake [3841]. Nevertheless, it has been shown that well-differentiated HCC can contain a considerable amount of Kupffer cells [40]. The high contrast between liver parenchyma and HCC in SPIO-enhanced MRI is particularly helpful 

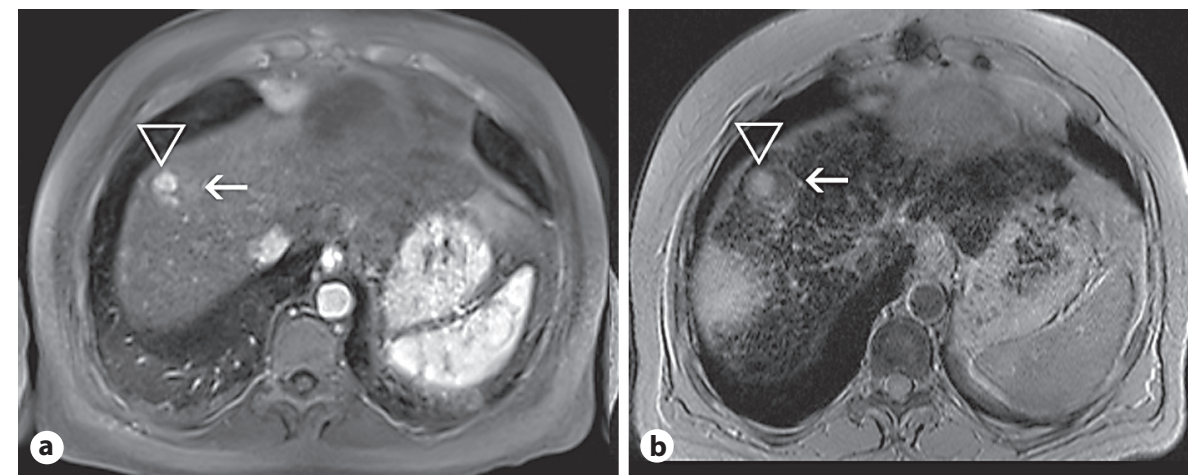

Fig. 7. A 56-year-old male patient with chronic hepatitis B and liver cirrhosis. Double-contrast MRI with a gadolinium-enhanced arterial phase $\mathrm{T}_{1} \mathrm{w} 3 \mathrm{D}$ GRE sequence (a) and a SPIO-enhanced $\mathrm{T}_{2} \mathrm{w}$ GRE sequence (b). Note the hypervascular spot (triangle) within the only slightly hyperenhancing nodule (arrow). The hypervascular spot returned to isointensity on portovenous phase images (not shown) and exhibits clearly missing SPIO uptake in the liver-specific phase (b), whereas the surrounding nodule shows preserved SPIO uptake. This lesion shows the so-called nodule-in-nodule appearance, representing an overt HCC (with typical vascular changes and missing SPIO uptake) which aroused in the surrounding regenerative nodule. for the evaluation of the tumor burden of the liver in case of the diffuse growth pattern of HCC. However, it is more or less accepted that evaluation of HCC without a multiphasic dynamic examination (either MDCT or gadolinium-enhanced MRI) is not sufficient since the assessment of pathological vascularity is still the main diagnostic criterion for HCC. Therefore, the combination of both SPIO and gadolinium contrast agents in one MR examination (so-called double-contrast technique) is considered as very useful $[39,42,43]$. In a recent study the detection rate and $\mathrm{Az}$ value of non-invasive double-contrast MRI was with 93 and $0.96 \%$, respectively, similar to combined CTAP/CTHA [44]. However, double-contrast MRI is offlabel use and it is a time-consuming examination with the need of two contrast agent injections (fig. 7).

With Mn-DPDP, HCC can typically be depicted as a hypointense lesion in the delayed-phase images. MnDPDP showed promising results with regard to the HCC detection. A study by Bartolozzi et al. [45] showed a detection rate of $86 \%$ for HCC, which was in this study slightly higher than with biphasic spiral CT (80\%, n.s.). Moreover, Mn-DPDP provides an additional criterion for the characterization of the different nodules in the cirrhotic liver [46]. However, because Mn-DPDP is not recommended for bolus injection, a second contrast agent has to be administered for the evaluation of the vascularity so that (like in double-contrast MRI) additional examination time and two contrast agent injections are needed. The two hepatobiliary contrast agents with also extracellular properties, Gd-BOPTA and Gd-EOB-DTPA, do not suf- fer from this limitation. They can be injected as a bolus and provide both a sufficient vascular phase as well as a hepatobiliary phase [47-49]. Their main difference is the dosing of gadolinium (Gd-EOB-DTPA: $0.025 \mathrm{mmol} / \mathrm{kg}$ b.w. compared to Gd-BOPTA $0.05 \mathrm{mmol} / \mathrm{kg}$ b.w.) and the higher amount of liver-specific uptake of Gd-EOB-DTPA (50 vs. $5 \%$ ) compared to Gd-BOPTA [48]. In a study by Kim et al. [44], Gd-BOPTA dynamic imaging (without delayed-phase imaging) yielded a mean sensitivity and positive predictive value of 91.3 and $89.2 \%$, respectively. A quite recent study with Gd-BOPTA showed excellent results for the detection of HCC up to values of $87 \%$ sensitivity and $79 \%$ specificity with a protocol including precontrast, early dynamic and 1-hour delayed images [50]. For the setting of the HCC and cirrhotic liver, little is known about the added value of the delayed phase for tumor detection - in contrast to metastases, where an increased detection rate was clearly documented with help of delayed-phase images [51]. Several reports have been published showing that missing hepatobiliary uptake of Gd-BOPTA is an additional criterion for HCC, especially in contrast to regenerative nodules [52-54]. Gd-EOBDTPA was approved in many countries worldwide between 2005 and 2008. Up to now there is still limited information about Gd-EOB-DTPA MRI in the cirrhotic liver. For a general population, Gd-EOB-DTPA showed (similar to Gd-BOPTA) an increased detection rate for malignant liver lesions compared to MDCT $[55,56]$. In the hepatobiliary phase of Gd-EOB-DTPA, almost all HCCs and some dysplastic nodules depict as an area of 
decreased liver-specific uptake resulting in hypointense lesions [57]. The early dynamic phase of Gd-EOB-DTPA was questioned due to the lower dosing of gadolinium; however, several reports documented that a sufficient arterial phase can be acquired and that hypervascular tumors can be depicted accurately $[58,59]$. However, due to the smaller injection volume it is recommended to use a bolus timing technique for Gd-EOB-DTPA-enhanced MRI, and there is data suggesting that a slower injection rate can lead to a more robust arterial phase with even increased arterial enhancement [60,61]. First emerging data from abstracts show promising results for Gd-EOBDTPA in the detection of HCC $[62,63]$. Also, the added value of the 20-min hepatobiliary-phase images has been documented by first abstracts. Lee et al. [64] demonstrated an increased rate of correctly diagnosed HCC from $65.2 \%$ for MDCT, $80.3 \%$ for early dynamic Gd-EOBDTPA, and $83.3 \%$ for combined dynamic and 20 -min hepatobiliary phase. In this study, $93.4 \%$ of HCCs showed to by hypointense in the hepatobiliary phase.

For all three hepatobiliary contrast agents, liver-specific uptake in malignant HCCs with depiction as hyperintense lesions in the liver-specific phase has been demonstrated, but usually the frequency of this finding is low $(<5 \%)$ and confined to well-differentiated HCCs [52, 57, $64,65]$. Generally, the presence of typical changes of the vascular supply will help to correctly characterize these lesions. Up to now, no exact thresholds for the uptake of regenerative nodules, dysplastic nodules and HCCs have been defined. However, the potential value of showing impaired biliary uptake for the evaluation of nodules in the cirrhotic liver has been appreciated by several authors $[13,66,67]$. In this respect it has been pointed out that hepatobiliary MRI helps to detect hepatic nodules 'at risk' for transformation into well-differentiated HCC (e.g. high-grade dysplastic nodules) prior to neovasculariza- tion and prior to development of overt HCC $[13,57]$. That implicates that in the future, for example nodules with features of a high-grade dysplasia at precontrast MRI ( $\mathrm{T}_{1} \mathrm{w}$ hyperintense, $\mathrm{T}_{2} \mathrm{~W}$ iso- or hyperintense) and with hypointense depiction in hepatobiliary-phase images might be considered as HCC from a certain size on even with missing neovascularization - helping to overcome the diagnostic gap which exists in hypovascular HCC [68]. However, such an approach still needs more data on large patient cohorts.

\section{Conclusion}

Although MDCT has reached a high standard for the detection of HCC with the possibility of multiphasic examinations and high-resolution isotropic datasets, MRI has to be regarded as the best non-invasive imaging modality for the detection of HCC and for the characterization of nodules in patients with liver cirrhosis. The multiple criteria (signal intensity precontrast, morphological features, early dynamic contrast-enhanced MRI, and especially liver-specific contrast agents) available with MRI result in a unique imaging modality enabling a very high diagnostic standard for the evaluation of the cirrhotic liver. However, it has to be recognized for all imaging modalities that there is a diagnostic problem for lesions $<1 \mathrm{~cm}$ (in the detection of these small HCC as well as in the differentiation from other non-neoplastic nodules). With regard to the guidelines by the EASL-AASLD [12], vascularity of lesions in the cirrhotic liver is still the main criterion; however, there is already good evidence that liver-specific contrast agents help to increase the detection rate and might overcome the diagnostic gap of hypovascular HCC. In this respect, larger prospective trials are wanted.

\section{References}

1 MacSween RNM, Burt A, Portmann B: Pathology of the Liver, ed 4. London, Churchill Livingstone, 2002.

2 Ferrell LD, Crawford JM, Dhillon AP, Scheuer PJ, Nakanuma Y: Proposal for standardized criteria for the diagnosis of benign, borderline, and malignant hepatocellular lesions arising in chronic advanced liver disease. Am J Surg Pathol 1993;17:1113-1123.

3 International Working Party: Terminology of nodular hepatocellular lesions. Hepatology 1995;22:983-993.
4 Matsui O: Imaging of multistep human hepatocarcinogenesis by CT during intra-arterial contrast injection. Intervirology 2004; 47:271-276.

5 Hwang GJ, Kim MJ, Yoo HS, Lee JT: Nodular hepatocellular carcinomas: detection with arterial-, portal-, and delayed-phase images at spiral CT. Radiology 1997;202:383-388.

6 Schima W, Kulinna C, Ba-Ssalamah A, Grünberger T: Multidetector computed tomography of the liver. Radiologe 2005; 45 : $15-23$.
7 Baron RL: Understanding and optimizing use of contrast material for CT of the liver. Am J Roentgenol 1994;163:323-331.

8 Kawata S, Murakami T, Kim T, et al: Multidetector CT: diagnostic impact of slice thickness on detection of hypervascular hepatocellular carcinoma. Am J Roentgenol 2002;179:61-66.

9 Haider MA, Amitai MM, Rappaport DC, et al: Multi-detector row helical CT in preoperative assessment of small $(<$ or $=1.5 \mathrm{~cm})$ liver metastases: is thinner collimation better? Radiology 2002;225:137-142. 
10 Wedegärtner U, Lorenzen $\mathrm{M}$, Nagel HD, et al: Image quality of thin- and thick-slice MSCT reconstructions in low-contrast objects (liver lesions) with equal doses. Rofo 2004; 176:1676-1682.

11 Iannaccone R, Laghi A, Catalano C, et al: Hepatocellular carcinoma: role of unenhanced and delayed-phase multi-detector row helical CT in patients with cirrhosis. Radiology 2005;234:460-467.

12 Bruix J, Sherman M: Management of hepatocellular carcinoma. Practice Guidelines Committee, American Association for the Study of Liver Diseases. Hepatology 2005;42: 1208-1236.

13 Bartolozzi C, Crocetti L, Lencioni R, Cioni D, Della Pina C, Campani D: Biliary and reticuloendothelial impairment in hepatocarcinogenesis: the diagnostic role of tissuespecific MR contrast media. Eur Radiol 2007;17:2519-2530.

14 Burrel M, Llovet JM, Ayuso C, et al: MRI angiography is superior to helical CT for detection of HCC prior to liver transplantation: an explant correlation. Hepatology 2003;38: 1034-1042.

15 Maetani YS, Ueda M, Haga H, et al: Hepatocellular carcinoma in patients undergoing living-donor liver transplantation. Accuracy of multidetector computed tomography by viewing images on digital monitors. Intervirology 2008;51(suppl 1):46-51.

16 Kim SJ, Kim SH, Lee J, et al: Ferucarbotranenhanced 3.0-T magnetic resonance imaging using parallel imaging technique compared with triple-phase multidetector row computed tomography for the preoperative detection of hepatocellular carcinoma. J Comput Assist Tomogr 2008;32:379-385.

17 Zech CJ, Herrmann KA, Huber A, et al: High-resolution MR-imaging of the liver with $\mathrm{T}_{2}$-weighted sequences using integrated parallel imaging: comparison of prospective motion correction and respiratory triggering. J Magn Reson Imaging 2004;20: 443-450.

18 Zech CJ, Schoenberg SO, Herrmann KA, et al: Modern visualization of the liver with MRT. Current trends and future perspectives. Radiologe 2004;44:1160-1169.

19 Rofsky NM, Lee VS, Laub G, et al: Abdominal MR imaging with a volumetric interpolated breath-hold examination. Radiology 1999;212:876-884.

20 Matsui O, Kadoya M, Kameyama T, et al: Adenomatous hyperplastic nodules in the cirrhotic liver: differentiation from hepatocellular carcinoma with MRimaging. Radiology 1989;173:123-126.

21 Kadoya M, Matsui O, Takashima T, Nonomura A: Hepatocellular carcinoma: correlation of MR imaging and histopathologic findings. Radiology 1992;183:819-825.

22 Shinmura R, Matsui O, Kobayashi S, et al: Cirrhotic nodules: association between MR imaging signal intensity and intranodular blood supply. Radiology 2005;237:512-519.
23 Hanna RF, Aguirre DA, Kased N, Emery SC, Peterson MR, Sirlin CB: Cirrhosis-associated hepatocellular nodules: correlation of histopathologic and MR imaging features. Radiographics 2008;28:747-769.

24 Grazioli L, Olivetti L, Fugazzola C, et al: The pseudocapsule in hepatocellular carcinoma: correlation between dynamic MR imaging and pathology. Eur Radiol 1999;9:62-67.

25 Onaya H, Itai Y: MR imaging of hepatocellular carcinoma. Magn Reson Imaging Clin N Am 2000;8:757-768.

26 Taouli B, Vilgrain V, Dumont E, Daire JL, Fan B, Menu Y: Evaluation of liver diffusion isotropy and characterization of focal hepatic lesions with two single-shot echo-planar MR imaging sequences: prospective study in 66 patients. Radiology 2003;226:71-78.

27 Zech CJ, Herrmann KA, Dietrich O, Horger W, Reiser MF, Schoenberg SO: Black-blood diffusion-weighted EPI acquisition of the liver with parallel imaging: comparison with a standard $\mathrm{T}_{2}$-weighted sequence for detection of focal liver lesions. Invest Radiol 2008; 43:261-266.

28 Xu PJ, Yan FH, Wang JH, Lin J, Ji Y: Added value of breathhold diffusion-weighted MRI in detection of small hepatocellular carcinoma lesions compared with dynamic contrast-enhanced MRI alone using receiver operating characteristic curve analysis. J Magn Reson Imaging 2009;29:341-349.

29 Taouli B, Chouli M, Martin AJ, Qayyum A, Coakley FV, Vilgrain V: Chronic hepatitis: role of diffusion-weighted imaging and diffusion tensor imaging for the diagnosis of liver fibrosis and inflammation. J Magn Reson Imaging 2008;28:89-95.

30 Lauenstein TC, Salman K, Morreira R, et al: Gadolinium-enhanced MRI for tumor surveillance before liver transplantation: center-based experience. Am J Roentgenol 2007; 189:663-670

31 Rode A, Bancel B, Douek P, et al: Small nodule detection in cirrhotic livers: evaluation with US, spiral CT, and MRI and correlation with pathologic examination of explanted liver. J Comput Assist Tomogr 2001;25:327336.

32 de Ledinghen V, Laharie D, Lecesne R, et al: Detection of nodules in liver cirrhosis: spiral computed tomography or magnetic resonance imaging? A prospective study of 88 nodules in 34 patients. Eur J Gastroenterol Hepatol 2002;14:159-165.

33 Stoker J, Romijn MG, de Man RA, et al: Prospective comparative study of spiral computer tomography and magnetic resonance imaging for detection of hepatocellular carcinoma. Gut 2002;51:105-107.

34 Kang BK, Lim JH, Kim SH, et al: Preoperative depiction of hepatocellular carcinoma: ferumoxides-enhanced MR imaging versus triple-phase helical CT. Radiology 2003;226: 79-85.
35 Brancatelli G, Baron RL, Peterson MS, Marsh W: Helical CT screening for hepatocellular carcinoma in patients with cirrhosis: frequency and causes of false-positive interpretation. Am J Roentgenol 2003;180:10071014.

36 Holland AE, Hecht EM, Hahn WY, et al: Importance of small $(<$ or $=20 \mathrm{~mm})$ enhancing lesions seen only during the hepatic arterial phase at MR imaging of the cirrhotic liver: evaluation and comparison with whole explanted liver. Radiology 2005;237:938-944.

37 Kim YK, Kim CS, Kwak HS, Lee JM: Threedimensional dynamic liver MR imaging using sensitivity encoding for detection of hepatocellular carcinomas: comparison with superparamagnetic iron oxide-enhanced MR imaging. J Magn Reson Imaging 2004; 20:826-837.

38 Yamamoto H, Yamashita Y, Takahashi M: Development of hepatomas in hyperplastic nodules induced in the rat liver: detection with superparamagnetic iron oxide-enhanced magnetic resonance imaging. Acad Radiol 1996;3:330-335.

39 Ward J, Guthrie JA, Scott DJ, et al: Hepatocellular carcinoma in the cirrhotic liver: double-contrast MR imaging for diagnosis. Radiology 2000;216:154-162.

40 Imai Y, Murakami T, Yoshida S, et al: Superparamagnetic iron oxide-enhanced magnetic resonance images of hepatocellular carcinoma: correlation with histological grading. Hepatology 2000;32:205-212.

41 Namkung S, Zech CJ, Helmberger T, Reiser MF, Schönberg SO: Superparamagnetic iron oxide (SPIO)-enhanced liver MRI with ferucarbotran: efficacy for characterization of focal liver lesions. J Magn Reson Imaging 2007;25:755-765.

42 Bhartia B, Ward J, Guthrie JA, Robinson PJ: Hepatocellular carcinoma in cirrhotic livers: double-contrast thin-section MRI with pathologic correlation of explanted tissue. AJR Am J Roentgenol 2003;180:577-584.

43 Kwak HS, Lee JM, Kim CS: Preoperative detection of hepatocellular carcinoma: comparison of combined contrast-enhanced MRI and combined CT during arterial portography and CT hepatic arteriography. Eur Radiol 2004; 14:447-457.

44 Kim YK, Kwak HS, Han YM, Kim CS: Usefulness of combining sequentially acquired gadobenate dimeglumine-enhanced magnetic resonance imaging and Resovist-enhanced magnetic resonance imaging for the detection of hepatocellular carcinoma: comparison with computed tomography hepatic arteriography and computed tomography arterioportography using 16-slice multidetector computed tomography. J Comput Assist Tomogr 2007;31:702-711.

45 Bartolozzi C, Donati F, Cioni D, Crocetti L, Lencioni R: MnDPDP-enhanced MRI vs. dual-phase spiral CT in the detection of hepatocellular carcinoma in cirrhosis. Eur Radiol 2000;10:1697-1702. 
46 Kim JH, Kim MJ, Park YN, et al: Mangafodipir trisodium-enhanced MRI of hepatocellular carcinoma: correlation with histological characteristics. Clin Radiol 2008;63: 1195-1204.

47 Vogl TJ, Stupavsky A, Pegios W, et al: Hepatocellular carcinoma: evaluation with dynamic and static gadobenate dimeglumineenhanced MR imaging and histopathologic correlation. Radiology 1997;205:721-728.

48 Reimer P, Schneider G, Schima W: Hepatobiliary contrast agents for contrast-enhanced MRI of the liver: properties, clinical development and applications. Eur Radiol 2004;14: 559-578.

49 Zech CJ, Herrmann KA, Reiser MF, Schoenberg SO: MR imaging in patients with suspected liver metastases: value of liver-specific contrast agent Gd-EOB-DTPA. Magn Reson Med Sci 2007;6:43-52.

50 Choi SH, Lee JM, Yu NC, et al: Hepatocellular carcinoma in liver transplantation candidates: detection with gadobenate dimeglumine-enhanced MRI. Am J Roentgenol 2008;191:529-536.

51 Kim YK, Lee JM, Kim CS, Chung GH, Kim CY, Kim IH: Detection of liver metastases: gadobenate dimeglumine-enhanced threedimensional dynamic phases and one-hour delayed-phase MR imaging versus superparamagnetic iron oxide-enhanced MR imaging. Eur Radiol 2005;15:220-228.

52 Kim JI, Lee JM, Choi JY, et al: The value of gadobenate dimeglumine-enhanced delayed-phase MR imaging for characterization of hepatocellular nodules in the cirrhotic liver. Invest Radiol 2008;43:202-210.

53 Grazioli L, Morana G, Caudana R, et al: Hepatocellular carcinoma: correlation between gadobenate dimeglumine-enhanced MRI and pathologic findings. Invest Radiol 2000; $35: 25-34$.
54 Tanimoto A, Kuwatsuru R, Kadoya M, et al: Evaluation of gadobenate dimeglumine in hepatocellular carcinoma: results from phase II and phase III clinical trials in Japan. J Magn Reson Imaging 1999;10:450-460.

55 Huppertz A, Balzer T, Blakeborough A, et al: Improved detection of focal liver lesions at MR imaging: multicenter comparison of gadoxetic acid-enhanced MR images with intraoperative findings. Radiology 2004; 230:266-275.

56 Hammerstingl R, Huppertz A, Breuer J, et al: Diagnostic efficacy of gadoxetic acid (Primovist)-enhanced MRI and spiral CT for a therapeutic strategy: comparison with intraoperative and histopathologic findings in focal liver lesions. Eur Radiol 2008;18:457467.

57 Saito K, Kotake F, Ito N, et al: Gd-EOBDTPA-enhanced MRI for hepatocellular carcinoma: quantitative evaluation of tumor enhancement in hepatobiliary phase. Magn Reson Med Sci 2005;4:1-9.

58 Huppertz A, Haraida S, Kraus A, et al: Enhancement of focal liver lesions at gadoxetic acid-enhanced MR imaging: correlation with histopathologic findings and spiral CT - initial observations. Radiology 2005; 234:468-478.

59 Zech CJ, Grazioli L, Breuer J, Reiser MF, Schoenberg SO: Diagnostic performance and description of morphological features of focal nodular hyperplasia in Gd-EOBDTPA-enhanced liver magnetic resonance imaging: results of a multicenter trial. Invest Radiol 2008;43:504-511.

60 Malone D, Zech CJ, Ayuso C, Bartolozzi C, Jonas E, Tanimoto A: Magnetic resonance imaging of the liver. Consensus statement from the 1st International Primovist User Meeting. Eur Radiol 2008;18(suppl 4):849864.
61 Zech CJ, Vos B, Nordell A, et al: Vascular enhancement in early dynamic liver MRI in an animal model: comparison of two injection regimen and two different doses Gd-EOBDTPA (gadoxetic acid) with standard GdDTPA. Inv Radiol 2009 (in press).

62 Di Martino M, Marin D, Guerrisi A, Geiger D, Galati F, Catalano C: Detection of hepatocellular carcinoma in patients with cirrhosis: intraindividual comparison of gadoxetic acid (Gd-EOB-DTPA)-enhanced MR imaging and multiphasic 64-slice CT. RSNA, 2008, p 282.

63 Lee JY, Kim SH, Kim SH, Jeon YH, Lee J, Kim MJ: Ferucarbotran-enhanced MRI versus gadoxetic acid-enhanced MRI for the preoperative detection of hepatocellular carcinoma. RSNA, 2008, p 670.

64 Lee JM, Kim SB, Lee JY, et al: Enhancement patterns on gadoxetic acid-enhanced MRI of hepatocellular carcinoma in the cirrhotic liver: comparison with multiphasic liver CT. 6th International Meeting Hepatocellular Carcinoma: Eastern and Western Experiences, Seoul, 2008, p 19.

65 Martí-Bonmatí L: MR imaging characteristics of hepatic tumors. Eur Radiol 1997;7: 249-258.

66 Kanematsu M, Kondo H, Goshima S, Tsuge $\mathrm{Y}$, Watanabe H: Magnetic resonance imaging of hepatocellular carcinoma. Oncology 2008;75(suppl 1):65-71.

67 Kudo M: Hepatocellular carcinoma 2009 and beyond: from the surveillance to molecular targeted therapy. Oncology 2008;75 (suppl 1):1-12.

68 Golfieri R, Coppola F, Fusco F, et al: Malignant progression of a small HCC nodule: hypovascular 'early HCC' converted to hypervascular 'small HCC' within six months. Dig Liver Dis 2007;39:883-890. 